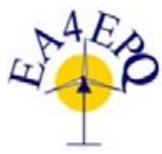

International Conference on Renewable Energies and Power Quality (ICREPQ'16)

Madrid (Spain), $4^{\text {th }}$ to $6^{\text {th }}$ May, 2016

Renewable Energy and Pamer Quality. Fourna(RE\&PQJ)

ISSN 2172-038 X, No.14 May 2016

\title{
Hidden schematics of EMI filters
}

\author{
M. Buzdugan, H. Balan \\ Technical University of Cluj-Napoca, \\ 28, Memorandumului str., Cluj-Napoca (Romania), \\ Phone number: +40 744560 833, Fax number: +40 264410 179, \\ e-mail: mircea.buzdugan@insta.utcluj.ro, \\ horia.balan@eps.utcluj.ro
}

\begin{abstract}
The paper presents the lumped parameters of EMI filters that must be used in the design process. In order to obtain consistent results, apart from the conventional parameters and stress de-rating factors (power dissipation, limiting voltage and current, operating temperature range, etc.), one should think also in terms of nonideal behaviour of the circuit elements in the equivalent model. Following this conduct the calculated filter characteristics (attenuation or insertion loss) will gain in accuracy.
\end{abstract}

\section{Key words}

EMI filter, differential mode, common mode, Bode plot, equivalent series resistance ESR, equivalent series inductance ESL, equivalent parallel capacity EPC.

\section{Introduction}

Electromagnetic interference (EMI) filters belong to the category of passive filters, being basically decision making circuits and let passing the energy of the useful signal, in general a low frequency one, and attenuate, stop, reflect or absorb the energy of the unwanted signals, in general high frequency ones which are assumed to be electromagnetic noise. Consequently EMI filters are almost always lowpass filters and are supposed to be effective in frequencies ranging from $100 \mathrm{kHz}$ to $30 \mathrm{MHz}$.

EMI filters design is quite a complicated task and therefore in literature are reported various low pass filter patterns [14]. However the language spoken between those dealing with standard filters and those dealing with EMI filters is totally different. In standard signal filters terms that poles, zeros, group delay, predistortion, attenuation and order of the filter are commonly used, while in EMI filtering terms like attenuation, insertion loss, filter voltage drop, filter voltage rise and number of filter sections necessary to meet a required insertion loss are used.

Some of the main reasons for the development and use of block mains filters are [5]:
- mandatory conducted emission standards concentrate on the mains port, hence there is an established market for filter units;

- add-on "fit and forget" filters can be retro-fitted;

- safety approvals for the filter have already been achieved;

- many equipment designers are not familiar with RF filter design.

A typical EMI filter includes components to block both common mode and differential mode components.

In differential mode the cable carries currents go and return, while in common mode currents are flowing in the same direction on each wire, generally having nothing at all to do with the useful currents. From Fig. 1 it can be observed that the differential mode currents and consequently the resulting electric fields are oppositely directed and will subtract to give a small net radiated field, which is not the case of common mode currents that have the same direction. One can easily conclude that common mode currents have a much greater potential in producing radiated emissions than differential mode currents.

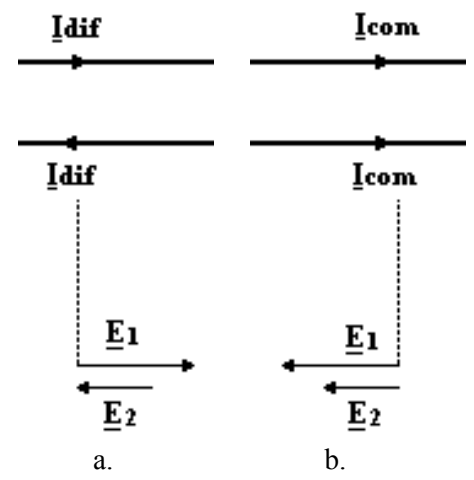

Fig. 1 The relative radiated emission potential of a. differential mode currents and $\mathrm{b}$. common mode currents

Fig. 2 presents a typical EMI mains filter. Capacitors $C_{Y 1}$ and $C_{Y 2}$ attenuate common mode interference and if $C_{X 2}$ is large, have no significant effect on differential mode. 
Capacitors $C_{X 1}$ and $C_{X 2}$ attenuate only the differential mode and can have fairly high values $(0.1$ to $0.47 \mu \mathrm{F}$ are typical ones).

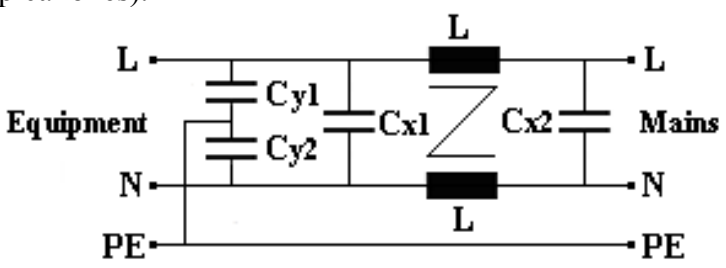

Fig. 2 Typical mains EMI filter

The common mode choke, which is the most important element of any EMI filter, presents a high value of the impedance for common mode signals and a low value of the impedance for the differential mode signals. It is built similarly to an inductor, but it is a so called a "two-wire product", which allows high inductance values, typically $1-10 \mathrm{mH}$, in a small volume without fear of choke saturation caused by the mains frequency supply current.

Since the windings of the common mode choke are identical and tightly wound on the same core, the mutual inductance is approximately equal to the self-inductance which means that the coupling coefficient is almost unity. Consequently the common-mode choke does affect differential-mode currents only by the value of the leakage inductance, due to the magnetic flux that leaks out the core and does not couple between the windings.

The differential and the common mode equivalent circuits of the EMI filters are depicted in Fig. 3 and Fig. 4 respectively.

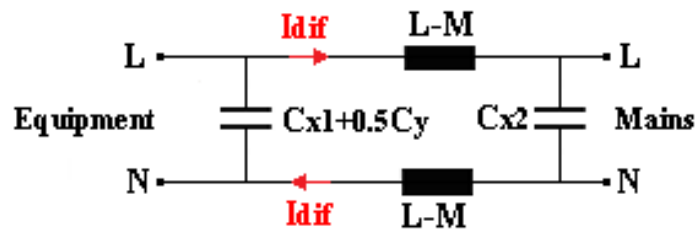

Fig. 3 The equivalent circuit of the differential mode filter

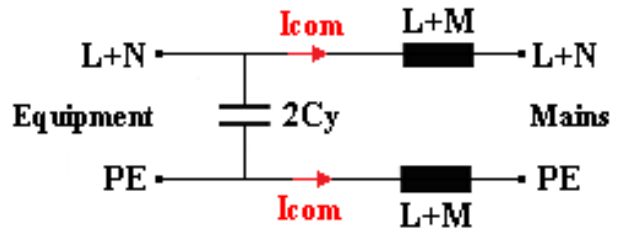

Fig. 4 The equivalent circuit of the common mode filter

Since EMI filters must operate adequately in the range of frequency from $100 \mathrm{kHz}$ to $30 \mathrm{MHz}$, any designer of EMI filters must think in terms of nonideal behavior of components and of their frequency response.

In order to obtain an adequate suppression of the conducted electromagnetic emissions it is crucially important to know the nonideal behavior of the components involved in EMI filters.

Consequently the following section will focus on the nonideal behavior of passive lumped components involved in EMI filters, which implies drawing so called "hidden schematics", containing the parasitic electrical parameters. The complexity of the EMI equivalent circuit models has an intermediary level between the ideal ones and the lumped elements used in microwave circuits [58].

\section{Nonideal behavior of EMI filters elements}

\section{A. Resistors}

A resistor value also depends on its frequency of operation. They have parasitic reactance, capacitive and inductive whose values vary versus frequency, affecting the resistance value and at a certain frequency giving rise even to self-resonance phenomena.

Figs. 5.a and b present two usual models valuable in a large range of frequency for a resistor.
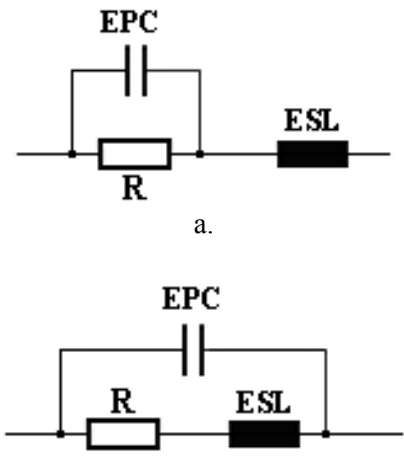

b.

Fig. 5 Equivalent schematics of a high frequency resistor

The equivalent inductance ESL in the models refers to the inductance of the loop area bounded by the two leads of the resistor. The parallel parasitic capacitance ECP refers to the parallel combination of the leads and leakage capacitances.

For the circuit presented in Fig. 5.a the Laplace transform of the impedance is:

$$
Z(s)=E S L \frac{s^{2}+\frac{s}{R \cdot E P C}+\frac{1}{E S L \cdot E P C}}{s+\frac{1}{R \cdot E P C}}
$$

and substituting $s$ with $j \omega$ :

$$
\underline{Z}(j \omega)=E S L \frac{-\omega^{2}+\frac{j \omega}{R \cdot E P C}+\frac{1}{E S L \cdot E P C}}{j \omega+\frac{1}{R \cdot E P C}}
$$

The theoretical corresponding Bode diagram is depicted in Fig. 6, in which $f_{1}=\frac{1}{2 \pi R \cdot E C P}$ is the first cut-off frequency and $f_{S}=\frac{1}{2 \pi \sqrt{E S L \cdot E P C}}$ the self resonant frequency $(\mathrm{SRF})$. 


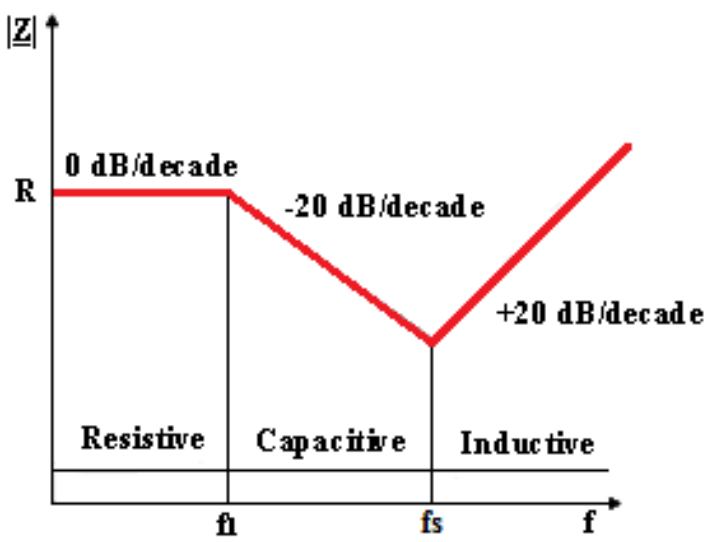

a.

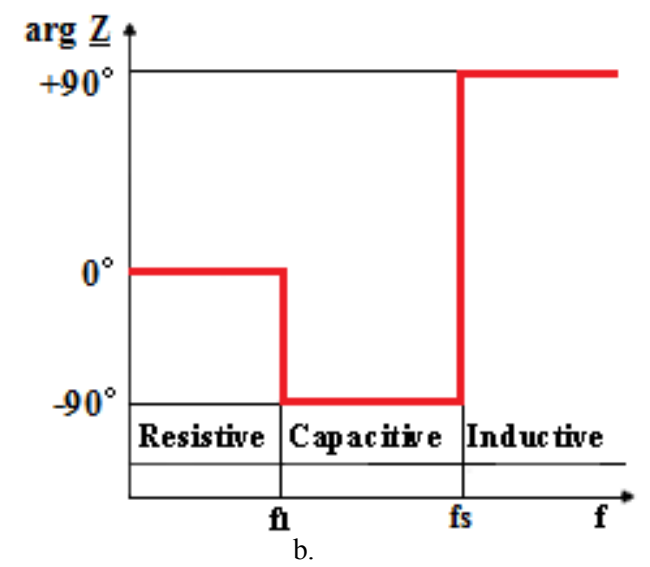

Fig. 6 Theoretical Bode plots of the impedance variation with frequency

The basic method is to plot not the magnitude $|\underline{Z}(j \omega)|$ but the logarithm of the magnitude

$$
|\underline{Z}(j \omega)|_{d B}=20 \log _{10}|\underline{Z}(j \omega)|(3)
$$

in decibels (above or relative to a reference level of $1 \mathrm{~V}$ ).

Fig. 7 presents the large range frequency behavior for a metallic resistor of $R=1.5 \mathrm{M} \Omega$, having the hidden parameters $E P C=1 \mathrm{pF}$ and $E S L=12 \mathrm{nH}$.

The Bode plots of the impedance variation with frequency are performed using Matlab.

One can easily see that the simulated example is quite close to the theoretical graph.

If the Bode plot is limited to the relevant range of conducted emissions, i.e. from $100 \mathrm{kHz}$ to $30 \mathrm{MHz}$ (see Fig. 8), one can see that the behavior of the resistor is acceptable from the point of view of the magnitude variation. The variation of the phase, which is relevant for conventional signal filters, is of slightly negligible importance in EMI filter design.

For the circuit presented in Fig. 5.b, the Laplace transform of the impedance is:

$$
Z(s)=\frac{s \cdot E S L+R}{s^{2} \cdot E S L \cdot E P C+s R \cdot E P C+1}
$$

and substituting $s$ with $j \omega$ :

$$
Z(j \omega)=\frac{j \omega \cdot E S L+R}{-\omega^{2} \cdot E S L \cdot E P C+j \omega R \cdot E P C+1}
$$

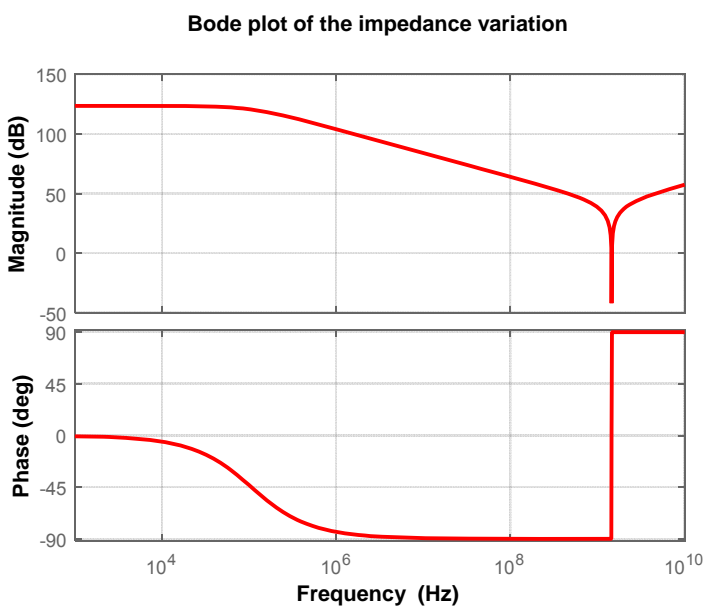

Fig. 7 Bode plots of the impedance variation with frequency of a resistor.

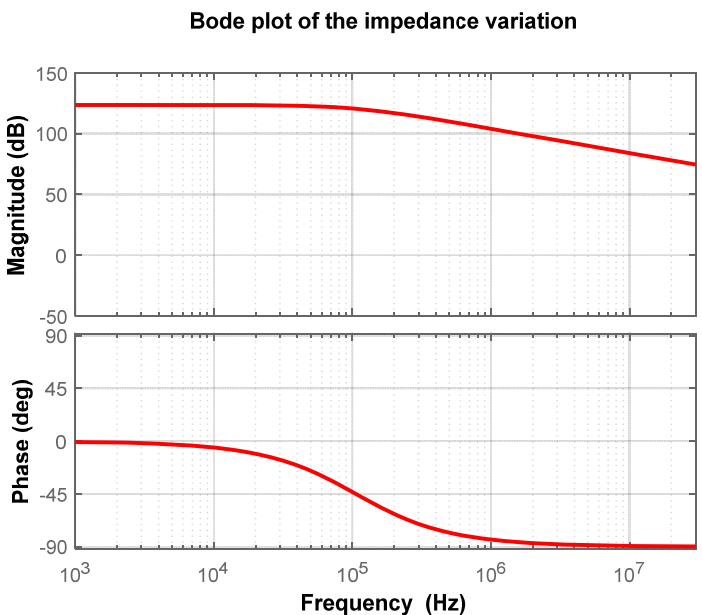

Fig. 8 Bode plots of the model from Fig. 5.a in the relevant range for conducted emissions

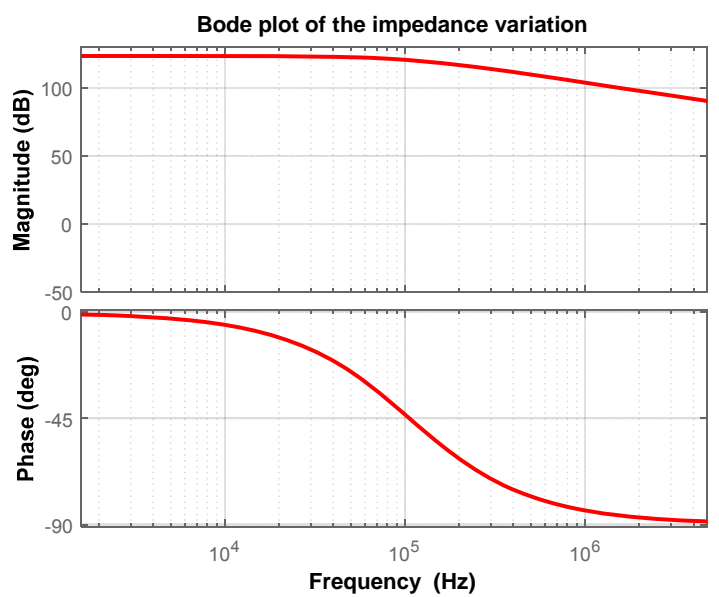

Fig. 9 Bode plots of the model from Fig. 5.b in the relevant range for conducted emissions

One can see that the behavior of the two models is the same in the range relevant for electromagnetic conducted 
emissions, but using the first model in microwave is not advisable.

\section{B. Capacitors}

All capacitors exhibit parasitic inductance due to their finite size and series resistance due to contact and electrode resistance. This series resistance is commonly known as ESR and is an important parameter in EMI filters design. Extra-low ESR values are contributing to a higher quality factor and a higher capability to store energy.

For the purposes of EMC suppression, the typical types of capacitors are the ceramic and the tantalum ones.

A lumped-element equivalent circuit of a capacitor is shown in Fig. 10, where ESL is the electrode inductance and EPC is the parasitic parallel capacitance and ESR is the resistance of the armatures.

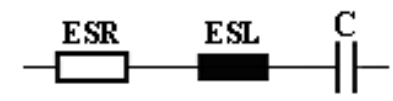

a.

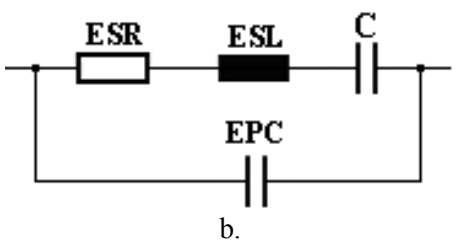

Fig. 10 Equivalent schematics of a high frequency capacitor

The impedance of the capacitance between the two electrodes can be written

$$
Z(s)=E S L \frac{s^{2}+\frac{s \cdot E S R}{E S L}+\frac{1}{E S L \cdot C}}{s}
$$

and substituting $s$ with $j \omega$ :

$$
\underline{Z}(j \omega)=E S L \frac{-\omega^{2}+\frac{j \omega \cdot E S R}{E S L}+\frac{1}{E S L \cdot C}}{j \omega}
$$

The theoretical Bode diagram (magnitude and phase) of the circuit is presented in Fig. 11, where $f_{S}=\frac{1}{2 \pi \sqrt{E S L \cdot C}}$ is the self resonance frequency, SRF.

As frequency increases, the impedance of the capacitor dominates but decreases linearly with frequency at a rate of $20 \mathrm{~dB} /$ decade while the impedance of the inductor increases until it equals that of the capacitor at the selfresonant frequency of the capacitor $f_{0}=\frac{1}{2 \pi \sqrt{L C}}$.

Beyond the self resonant frequency the inductor dominates, thus when a capacitor is used for shunting noise currents to ground, the frequency of the current must be lower that the self resonant frequency of the capacitor; that must be a rule of thumb for the designer.
Fig. 12 depicts the behavior of a ceramic capacitor in a large range of frequency, having $C=150 \mathrm{nF}, E S L=420$ $\mu \mathrm{H}, E S R=0.1 \Omega$.
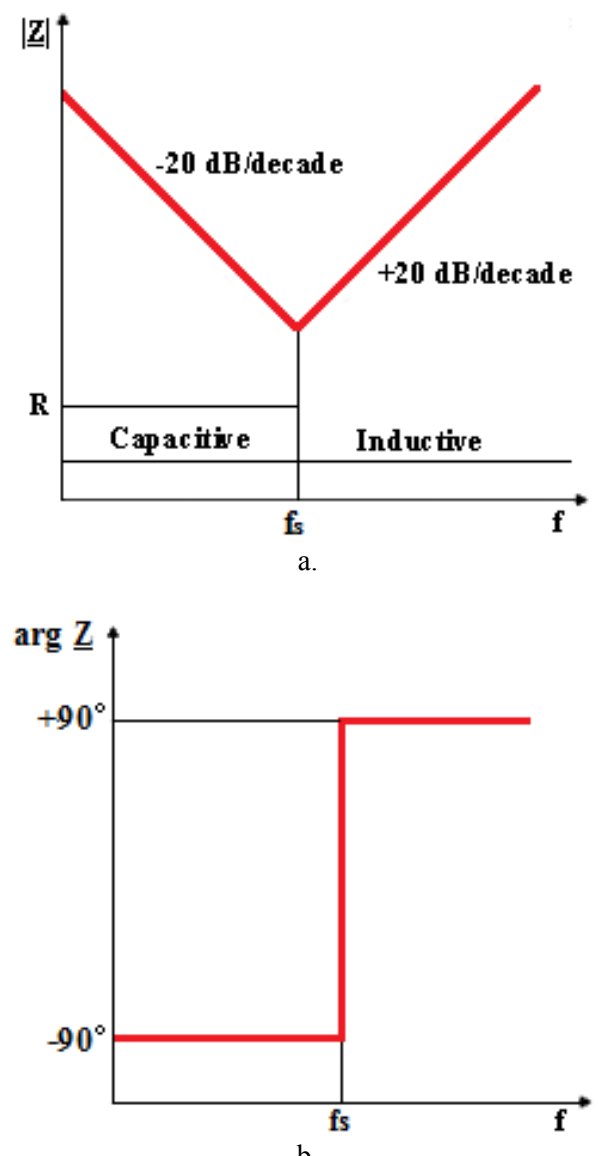

b.

Fig. 11 Theoretical Bode plots of the impedance variation with frequency of a capacitor.

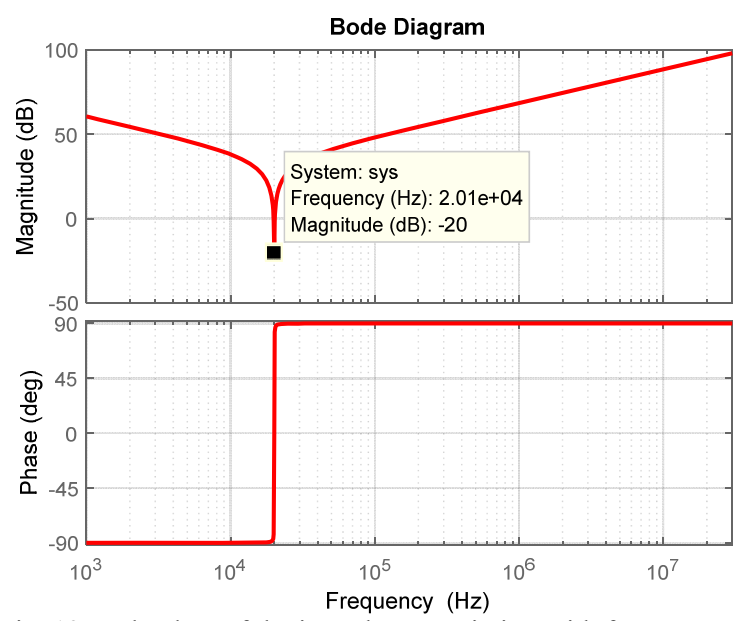

Fig. 12 Bode plots of the impedance variation with frequency of the capacitor from Fig. 8.a

Another lumped-element equivalent circuit model of a capacitor is shown in Fig. 10.b, where ESL is the electrode inductance and EPC is the parasitic parallel capacitance. It exhibits both series and parallel resonance frequencies where the series and first parallel resonance frequencies are dominant. 
Below the series resonance frequency $\left(\omega_{s} \cdot E S L=\frac{1}{\omega_{s} C}\right)$, the capacitor works as a capacitor as designed. However, above the resonance frequency, the capacitor's total reactance is inductive and it becomes again capacitive after the first parallel resonance frequency

$\left(\omega_{p} \cdot E S L=\frac{1}{\omega_{p} \cdot E P C}\right)$.

The impedance of the capacitor can be written

$$
Z(s)=\frac{1}{s \cdot E P C+\left(E S R+s \cdot E S L+\frac{1}{s C}\right)^{-1}}
$$

And substituting $s$ with $j \omega$

$$
\underline{Z}(j \omega)=\frac{1}{j \omega \cdot E P C+\left(E S R+j \omega \cdot E S L+\frac{1}{j \omega C}\right)^{-1}}
$$

Considering $\mathrm{EPC}=1.2 \mathrm{pF}$ the behavior of the equivalent model of the capacitor from Fig. 10.b in the range of frequency from $100 \mathrm{kHz}$ to $30 \mathrm{MHz}$ is plotted in Fig. 13. One can see at $7 \mathrm{MHz}$ an angular point of the graph, determining an uncontrolled increase of the impedance and another change in the phase.

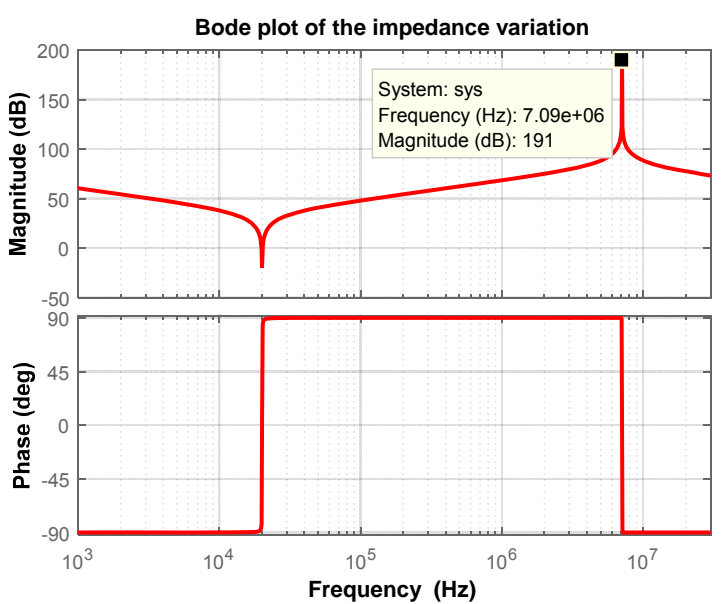

Fig. 13 Bode plots of the impedance variation with frequency of the capacitor from Fig. 10.b

\section{Inductors}

The specificities of the inductors' construction determine the parasitic elements in the EC model, due basically to the intrinsic resistance and the inter-turns and inter-layers capacities.

In literature are reported the equivalent circuit models depicted in Fig. 14.a and b.

The impedance of the model in Fig. 14.a is:

$$
Z_{L}(s)=E S R \frac{\frac{s L}{E S R}+1}{s^{2} L \cdot E P C+s \cdot E S R \cdot E P C+1}
$$

and substituting $s$ with $j \omega$ :

$$
\underline{Z}_{L}(j \omega)=E S R \frac{\frac{j \omega L}{E S R}+1}{-\omega^{2} L \cdot E P C+j \omega \cdot E S R \cdot E P C+1}
$$

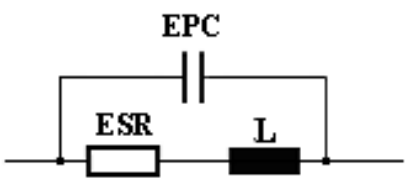

a.

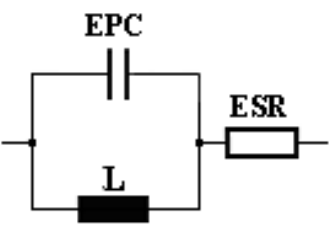

b.

Fig. 14 Equivalent schematics of a high frequency inductor

From Fig. 15 one can observe that at low frequencies, till $f_{1}=\frac{E S R}{2 \pi L}$ the resistance ESR is dominant. As frequency increases, the inductance dominates along with the decrease of the parasitic capacitance, until reaching the self resonant frequency. Beyond the self resonant frequency the capacitance is the one that dominates.

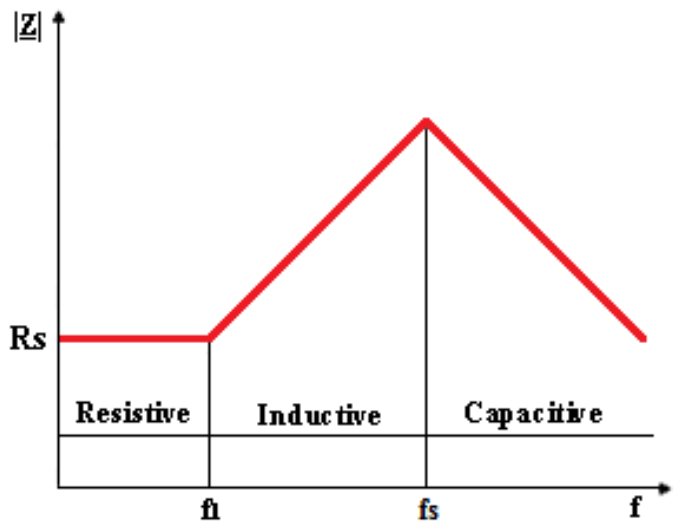

a.

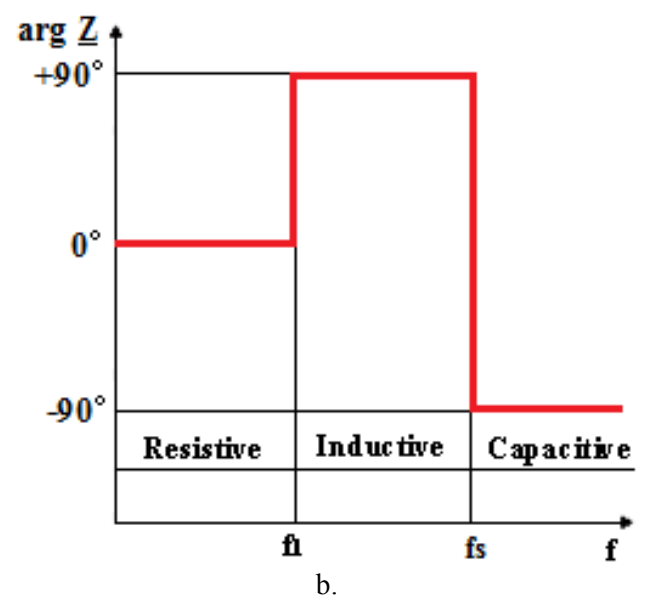

Fig. 15 Theoretical Bode plots of the impedance variation with frequency of an inductor 


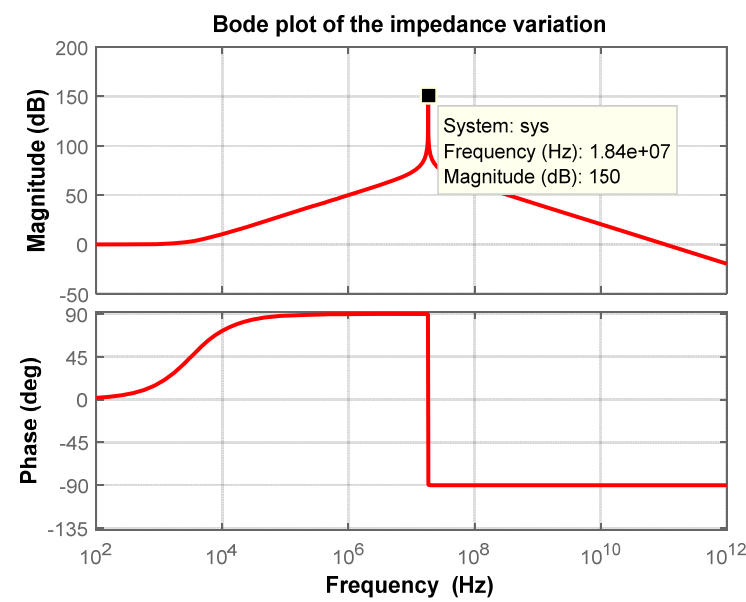

Fig. 16 Bode plots of the impedance variation with frequency of the inductor from Fig. 14.a

The Bode plot of the equivalent model from Fig. 14.a is shown in Fig. 16 for an inductor having the following parameters: $L=50 \mu \mathrm{H}, E S R=1 \Omega$ and $E P C=1.5 \mathrm{pF}$. One can see that the self resonance frequency, i.e. $18 \cdot 10^{6} \mathrm{~Hz}$, is at the superior margin, the range of standard conducted electromagnetic emissions (i.e. $30 \mathrm{MHz}$ ).

The impedance of the model in Fig. 14.b is:

$$
Z(s)=\left(s \cdot E P C+\frac{1}{s L}\right)^{-1}+E S R
$$

and substituting $s$ with $j \omega$ :

$$
Z(j \omega)=\left(j \omega \cdot E P C+\frac{1}{j \omega L}\right)^{-1}+E S R
$$

The Bode plot of the equivalent model from Fig. 14.b is shown in Fig. 17.

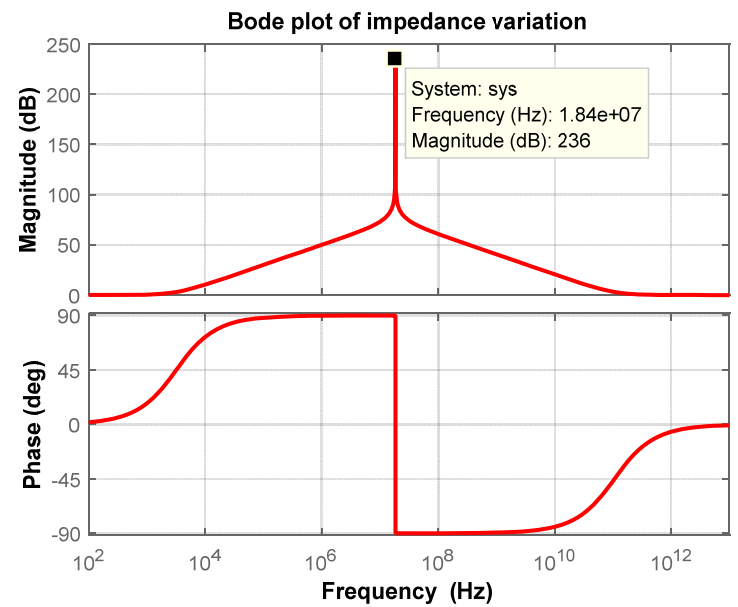

Fig. 17 Bode plots of the impedance variation with frequency of the inductor from Fig. 14.b

The self resonant frequency is the same as in Fig. 16, but the amplitude of the resonance is much higher in magnitude.

\section{Conclusion}

Parallel mounted capacitors are used in electromagnetic compatibility to divert noise currents, while series mounted inductors block noise currents. The option for parallel mounted capacitors or for series mounted inductors depends on the load impedance. If the load impedance presents a high inductive component, the option is for parallel mounted capacitors, blocking inductors being more effective in circuits having low values of the load impedance.

At the same time one must keep in mind that increasing the values of the inductance of an inductor will not determine necessarily lower impedance at higher frequencies, but will only decrease the self resonant frequency of the inductor.

The same observation is valuable in the case of the capacitors.

However some of the limitations of the passive filters are apparent and can be summarized as follows [9]: they are not adaptable to the changing system conditions, the passive elements in the filters are close tolerance components, the design is largely affected by the system impedance and often require a number of parallel shunt branches.

\section{References}

[1] Yu Zhenyang; Wang Shishan; Song Zheng; Bor-Lin Lee, "The reviews of integrated EMI filters applied in power electronic system", Asia-Pacific Symposium on Electromagnetic Compatibility, May 2015, pp. 227-230

[2] Qingbin Chen; Wei Chen; Subin Lin; Jiqing Dong, "The analysis and application of the near-field characteristics in the EMI filter", International Electronics and Application Conference (PEAC), Nov. 2014, pp 600 - 604

[3] I. Kovacevic, A. Muesing, T. Friedli, J.W. Kolar, "Electromagnetic Modeling of EMI Input Filters", $7^{\text {th }}$ International Conference on Integrated Power Electronics Systems, 2012, pp. 1-9

[4] R. L. Ozenbaugh, EMI Filter Design, Marcel Dekker, Inc. (2001), ISBN: 0-8247-8924-5, pp. 1-6

[5] T. Williams, EMC for Product Designers, Elsevier Ltd. (2007), ISBN: 978-0-7506-8170-4, pp. 340-380

[6] M. Buzdugan, H. Balan, "EMI filtering of air handling units", International Conference CIBv, Braşov, România Oct. 2015.

[7] C. R. Paul, Introduction to electromagnetic compatibility, John Wiley \& Sons (2006), ISBN-13: 978-0-471-75500-5, pp. 299-416

[8] I. Bahl, Lumped Elements for RF and Microwave Circuits, Artech House (2003), ISBN 1-58053-309-4, pp. 268-280,

[9] J. C. Das, Power system harmonics and passive filter design, John Wiley \& Sons, Inc. (2015), ISBN 978-1-118-86162-2, pp. 734-735 